Historic, archived document

Do not assume content reflects current scientific knowledge, policies, or practices. 



\section{The WESTMINSTER NURSERY}

J. E. STONER, Proprietor

WESTMINSTER, MARYLAND

\section{Planter's Special Price List---For Fall 1924}
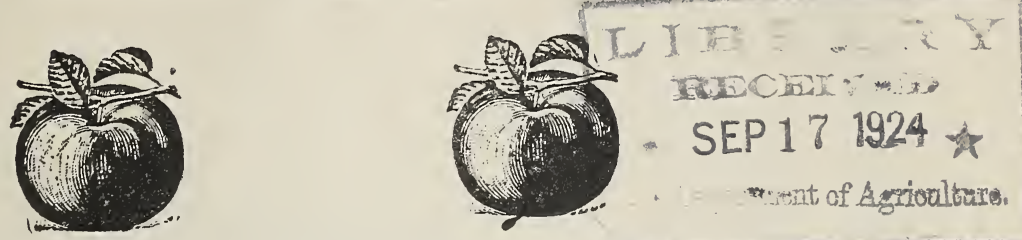

To our mail order trade:

We have a few facts to tell you, backed up by our millions of choice, healthy, vigorous, well grown and graded Peach, Apple, Pear, Cherry and Plum trees, also Berry plants, Califorma Privets, Barberry Thunbergi, Evergreen Shrubbery, anà Asparagus plants, etc. price.

Our stock has made a fine growth. You can't get better anywhere at any

The best is the cheapest, is the verdict of old, experienced orchardists-profit by facts established by others. We ship direct. We are the lowest priced Nursery on earth for sterling quality. We attach Entomologists certificates to all shipments.

Nothing you can grow will give you the permanent income that comes from an orchard.

Place your orders now. Make sure of your trees. Name kinds, grade and number wanted. We will reserve the trees until you want them shipped.

Special Discount-We will allow a special discount of 5 per cent on all cash orders received on or before Oct. 1st; after that date 3 per cent discount for Fall shipment. 5 per cent discount before March 1st; after that date 3 per cent dis. count for Spring shipment.

Boxing and Packing Free-We box and pack all orders without charge. All orders are put up in boxes or bales which are lined with heavy paper to guard against stock drying out. The trees and plants are packed in moist excelsior and straw. This is expensive, but good packing pays-pays customers, pays us. W. guarantee the arrival of stock in good condition.

Freight and Express Rates-Do not be influenced by the story of the agent who says that the freight or express on your order will bring our price up as high as his. If you will consult your railroad agent you will find that the freight rates frcm Westminster to points east of the Mississippi range from 20 to 40 cents per 100 pounds. First class trees will average about one pound each. Therefore, at the highest rate, 40 cents for 100 pounds, it means that you pay less than one-half cent per tree for freight. Nursery stock is carried by all express companies at a discuunt of 20 per cent less than merchandise rates.

Misrepresentation of the cost of getting stock from us by freight or express is sometimes made by others. Remember, if you bought your stock delivered, or freight prepaid, the freight would be included in the purchase price. The cash discounts which we allow will, in many cases, more than cover your freight or express charges.

Grading-We guarantee every tree to be up to the grade specified, or better. Many times trees are sold by height only. This, we believe, to be very misleading as we have seen trees which belonged to the second or third grade (if graded by size and calibre) sold for first grade, as they had the required height. We grade and sell our trees both by height and calibre, and believe this to be the only way that gives entire satisfaction to the planter.

Location-Westminster is located twentyl eight miles northwest of Baltimore, on the main line of the Western Maryland R. R., running from Baltimore to Pitts. burgh, which gives us good shipping facilities.

Our Soil is a deep limestone and rolling, and our nursery grounds lay high, which is just the kind to grow plenty of fibrous roots and form a good stem that will support a tree when it is transplanted into the soil. 


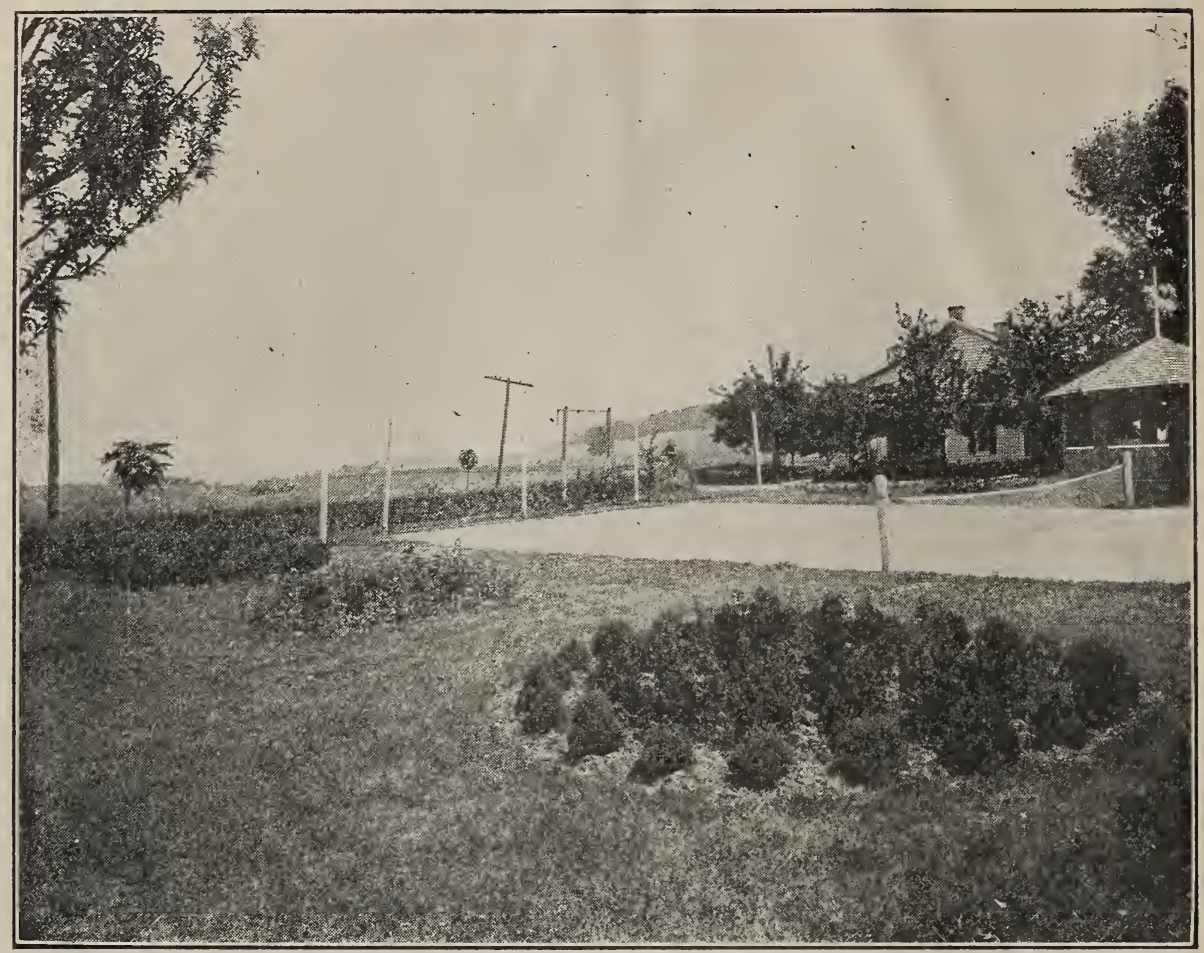

This shows part of the Home Grounds of J. E. Stoner's residence. You ean at a very nominal cost beantify your home with Evergreens, Cal. Privets, Boxwood, Etc. Plotographed Juily 10, 1924.

Special Prices on Large Orders, Special quotations on large orders will be given on application. When writing, tell us what varieties are wanted, the approximate number of trees of each variety, and the size.

Can't you arrange to visit our nursery and see how we grow our Stock?

NOTE:-1 to 4 Trees or Plants of a kind are sold at "Each" rates; 5 to 49 are sold at the " 10 " rates; 50 to 299 are sold at the " 100 " rate; 300 are sold at the " 500 ", and " 1000 "' rates.

All stock nicely packed F. O. B. cars here, in good boxes or bales at prices named in price list.

TERMS:-Orders from parties without rating, billed eash before shipment or one-quarter cash with order, balance when stock is shipped. If all cash with order you get the discount. No discount un'ess all cash with order. This price list is our salesman, and opens the way for every planter to secure our sterling quality trees at the lowest possible prices.

\section{APPLES : The following varieties of Apple Trees at prices below:}

$\begin{array}{rrrr}\text { Each } & 10 & 100 & 500\end{array}$

2 year, 5 to 7 feet, $11-16$ inch and up in caliper $\mathrm{XXX} \quad .70 \quad \$ 6.00 \quad \$ 50.00 \quad \$ 225.00$

2 year, 4 1-2 to 6 feet, 5-8 to 11-16 inch caliper XX. . $.60 \quad 4.50 \quad 40.00 \quad 175.00$

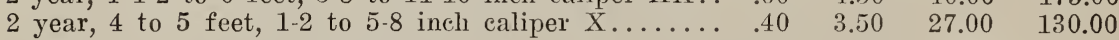

\begin{tabular}{|c|c|c|c|c|}
\hline APPLE ONE YEAR TREES: & Each & 10 & 100 & 1000 \\
\hline 5 to 6 feet... & .50 & $\$ 4.50$ & $\$ 40.00$ & $\$ 350.00$ \\
\hline 4 to 5 feet & .40 & 3.50 & 30.00 & 27 \\
\hline to 4 feet & .35 & 3.00 & 25.00 & 20 \\
\hline to 3 feet & .25 & 2.00 & 16.00 & 135.00 \\
\hline
\end{tabular}




Aken Red
Baldwin
Ben Davis
Bismarck
Black Ben Davis
Duchess
Delicious
Early Harvest
Early Ripe
Fall-Water
Gano
Gravestein
Golden Sweet

\section{A PARTIAI IIST OF VARIETIES}

Grimes Golden
Jonathan
King
King David
Liveland Raspberry
Maiden's Blush
McIntosh
N. W. Greening
Opalescent
Paradise
Red Astrachan
Rome Beauty
Stark

Smoke House

Sümmer Rambo

Spitzenburg

Stayman's Wine Sap

Winter Banana

Wm. Early Red

Wine Sap

Wealthy

Wagener

Walfriver

Yellow Transparent

York Imperial, Etc.

Add $5 \mathrm{e}$ each per tree to the above prices for the following varieties:

$\begin{array}{llll}\text { Duchess } & \text { MeIntosh } & \text { Grimes Golden } & \text { Baldwin } \\ \text { Wealthy } & \text { Jonathan } & \text { Rome Beauty } & \end{array}$

Parcel Post Size 2 to $3 \mathrm{ft}$. and 3 to $4 \mathrm{ft}$. only at $5 \mathrm{c}$ extra per tree.

\section{PEACHES :}

PRICES OF ALL VARIETIES OF PEACH TREES-1 year from Bud

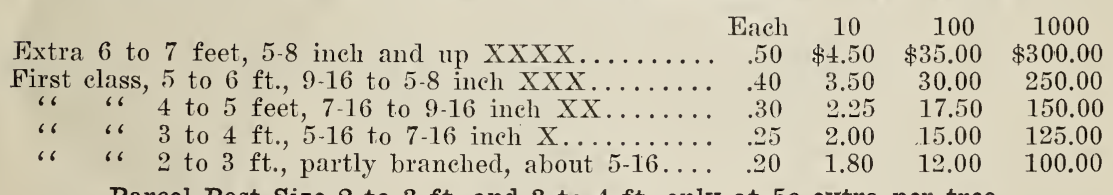

Parcel Post Size 2 to $3 \mathrm{ft}$. and 3 to $4 \mathrm{ft}$. only at $5 \mathrm{c}$ extra per tree.

Our Peach Trees have made an excellent growth and will grade up fine and we have a good supply to offer. Mostly 5 to $6 \mathrm{ft}$., 4 to $5 \mathrm{ft}$. and 3 to $4 \mathrm{ft}$. trees. Nice trees. A large stock of Elberta.

I wish to call your special attention to the fact that every one of our large stock of peach trees are budded on Kansas and North Carolina Natural seedlings, the most hardy in the U. S. You cannot afford to overlook this important feature. Our trees are well grown, smooth and well rooted. Our prices are low, considering the quality. Let us have your order at once that we may reserve the varieties for you. Notice about time of Ripening for Westminster, Md.

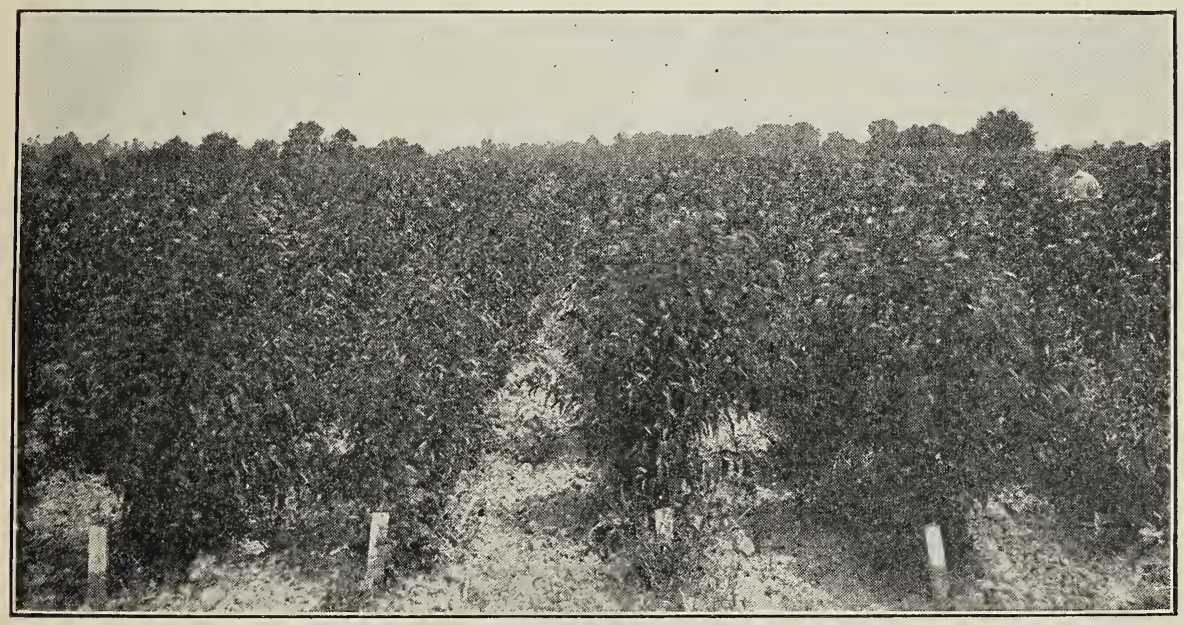

This is one block of about 60,000 Peach Trees coming 1 year from buds. Note excellent growth. Photographed July 10, 1924.

5 per cent discount on all cash orders received on or before Oct. 1st; after that date 3 per cent discount for Fall shipment. 5 per cent discount before March 1st; after that date 3 per cent discount for Spring shipment. 


\section{A PARTIAL LIST OF VARIETIES}

Alten (Aug. 1 to 10)

Bilyeau (Oct.)

Belle of Georg:a (Ea. Aug.)

Crummels Late (Oct.)

Champion (Aug. 25)

Chair's Choice (Sept. 12)

Crawford's Late (Sept. 18)

Carman (Aug. 5)

Captain Ede (Sept. 5)

Elberta (Sept. 1)

MeCallister (Sept. 15)

Matthews Beauty (Aug. 20)

Mayflower (July 1)

Mt. Rose (Aug. 15)

Niagara (Sept. 10)

O. M. Free (Sept. 15)

Picquet's Late (Sept. 28)

Ray (Aug. 10)

Reeve's Favorite (Sept. 12)

Roberta (Sept. 6th to 10th)

\author{
Rochester \\ Smock Beers (Sept. 25) \\ Fox Seedling (Sept. 15) \\ Ford's Late White (Sept. 20) \\ Globe (Sept. 28) \\ Geary Hold On (Sept. 10) \\ Greensboro (July 4) \\ J. H. Hale (Sept. 10) \\ Hiley Early (July 20) \\ Iron Mountain (Sept. 25) \\ Late Stump (Sept. 28) \\ Morris White (Sept. 30) \\ Stump (Sept. 20) \\ Slappey (July) \\ Salway (Sept. 30) \\ Willett (Sept. 10) \\ White Heath Cling (Sept. 28) \\ Wheatland (Sept. 25) \\ Yellow St. John (July 25)
}

Add 5e per tree extra

\section{PRICES OF ALL VARIETIES OF PEAR TREES STANDARD PEARS}

My pears are grown on imported French stock. Price of Pear Trees, 2 Years

\begin{tabular}{|c|c|c|c|c|c|}
\hline & & & Each & 10 & 100 \\
\hline irst & Class, & extra, 5 to 7 feet, $3-4$ inch and up $X X X$. & $\$ 1.00$ & $\$ 9.00$ & $\$ \mathcal{E} 5.00$ \\
\hline & & X.... & 8. & 8.00 & \\
\hline & ، & 4 to 5 Ieet, $1-2$ to $5-8$ inch 2 & .75 & $\begin{array}{l}6.50 \\
6.00\end{array}$ & $\begin{array}{l}50.00 \\
45.00\end{array}$ \\
\hline
\end{tabular}

Special Prices will be given for orders of 125 or more.

Parcel Post Size 3 to $4 \mathrm{ft}$. add $5 \mathrm{c}$ per tree extra.

\section{PRICES OF ALI VARIETIES OF DW ARF PEAR TREES}

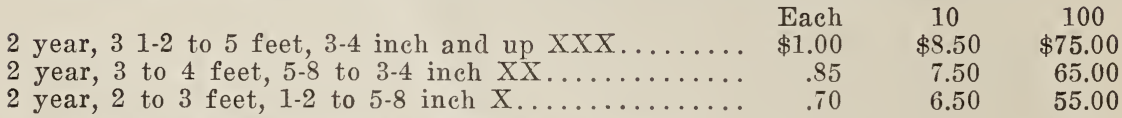

Add 2c per tree extra for Bartlett and Sickle.

Parcel Post Size 2 to $3 \mathrm{ft}$. and 3 to $4 \mathrm{ft}$. add je per tree extra.

\section{VARIETIES OF STANDARD AND DWARF PEARS}

$\begin{array}{lll}\text { Bartlett } & \text { Flemish Beauty } & \text { Sheldon } \\ \text { Clapp's Favorite } & \text { Kieffer } & \text { Sickle } \\ \text { Duchess } & \text { Lawrence } & \text { Wilder, Etc. }\end{array}$

\section{PRICES OF ALL VARIETIES OF CHERRY TREES}

\begin{tabular}{|c|c|c|c|}
\hline & Each & 10 & \\
\hline 2 year, 5 to 7 feet & 1.40 & $\$ 12.00$ & $\$ 100.00$ \\
\hline 2 year, $41-2$ to 6 feet, $5-8$ to $3-4$ inch $X X$ & 1.10 & 9.00 & 80. \\
\hline year, 4 to 5 feet, $1-2$ to $5-8$ inch $X \ldots \ldots$ & .85 & 7.50 & 70. \\
\hline ar, 3 to 4 feet.......... & .75 & 6.50 & \\
\hline
\end{tabular}

Parcel Post Size 3 to $4 \mathrm{ft}$. add $5 \mathrm{c}$ per tree extra.

\section{VARIETIES} ency.

Sour and Duke's Early Richmond, Dye House, Olivet and Large Montmor-

Sweets, White Ox Heart, Governor Wood, Napoleon, Ida, Black Tartarian, Windsor, Yellow Spanish, Bing, Ete. 
CRAB APPLES

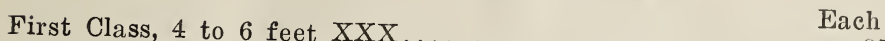

"6 Class, 4 to 6 feet XXX.......................

Varieties, Hyslop, Martha, Whitney and Transcendent.

Per 10

$\$ 7.00$

5.00

\section{APRICOTS}

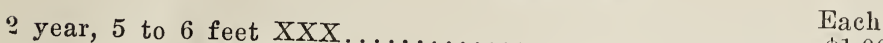

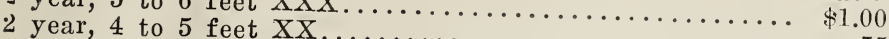

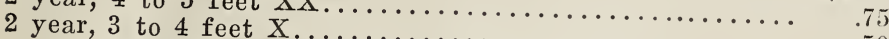

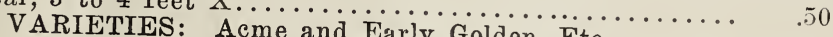

Per 10

$\$ 9.00$

7.00

4.50

Parcel Post Size 3 to $4 \mathrm{ft}$. add $5 \mathrm{c}$ per tree extra.

\section{PRICES OF ALL VARIETIES OF PLUM TREES}

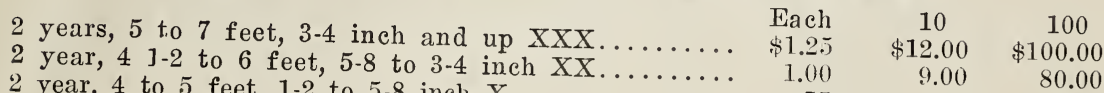

2 year, 4 to 5 feet, $1-2$ to $5-8$ inch X............. $\begin{array}{rrr}.75 & 9.00 & 80.00\end{array}$

\begin{tabular}{lll} 
Abundance & \multicolumn{1}{c}{ VARIETIE:S } \\
Bradshaw & Burbank & Satsuma \\
Red June & Shropshire Damson & Wickson \\
German Prune & Moore's Aretic & Lombard \\
\hline & Climax & Gold, Etc.
\end{tabular}

QUINCE

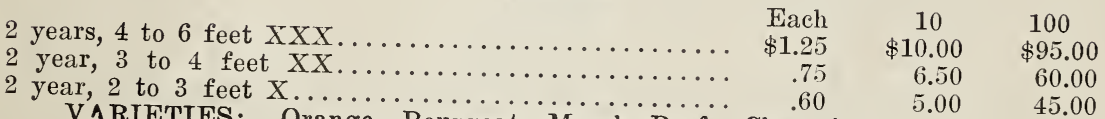

V.1RIETIES: Orange, Bourgeat, Meech Prof., Champion.

Parcel Post Size 2 to $3 \mathrm{ft}$. add 5e per tree extra.

RASPBERRY

Cumberland (Black)

Price Per 10 Per 100 Per 1000

Kansas and Eureka (B.................................... $\$ 4.00 \quad \$ 25.00$

Ruby and

St Rigs Everbearing $($........................... $.60 \quad 3.50 \quad 25.00$

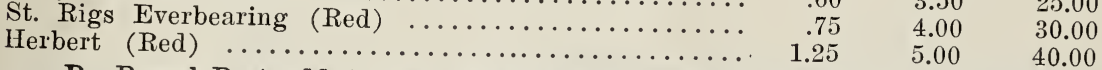
tities.

By Parcel Post add 1c per plant extra. Special prices given on large quan:

\section{BLACKBERRIES}

Price Each Per 10 Per 100 Per 1000

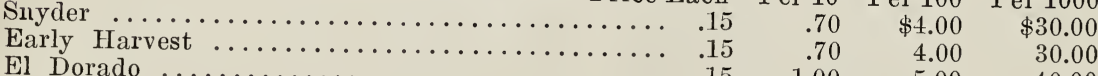

Blower ................................. $15 \quad 1.00 \quad 5.00 \quad 40.00$

$\begin{array}{lllll} & 15 & 1.00 & 5.50 & 35.00\end{array}$

$\begin{array}{lrrrr}\cdots & .10 & .60 & 4.00 & 30.00\end{array}$

MULBERRY

Downing Hicks, Price Each Per 10

$\$ 10.00$

CURRANTS

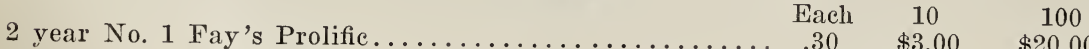

1 year No. 1 Fay's Prolific..................................... $\$ 3.00 \quad \$ 20.00$

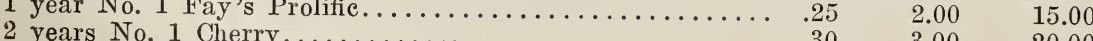

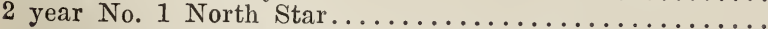

By Parcel Post add 2c per plant extra. 


\section{GOOSEBERRIES}

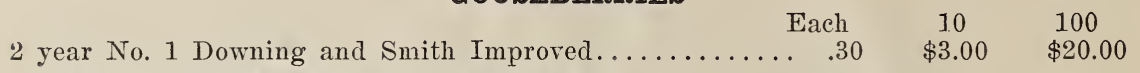

By Parcel Post add 2c per plant extra.

\section{GRAPES}

BLACK VARIETIES:

\begin{tabular}{ccrr} 
Each & 10 & 100 & \multicolumn{1}{c}{1000} \\
.30 & $\$ 2.50$ & $\$ 20.00$ & $\$ 150.00$ \\
.25 & 2.00 & 15.00 & 125.00 \\
.25 & 1.75 & 12.00 & 100.00 \\
.20 & 1.50 & 10.00 & 75.00 \\
.30 & 2.25 & 18.00 & 150.00 \\
.25 & 2.00 & 15.00 & 125.00
\end{tabular}

2 year No. 1 Moores' Early............................ $\$ 2.50 \quad \$ 20.00 \quad \$ 150.00$

1 year No. 1 Moores' Early........................ .25 $2.00 \quad 15.00 \quad 125.00$

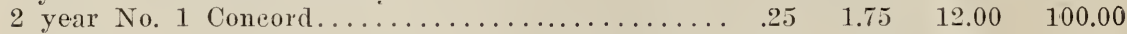

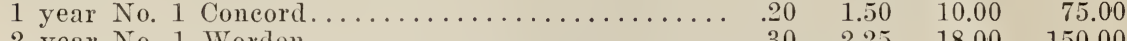

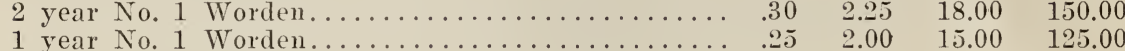

RED VARIETIES:

Each $\quad 10 \quad 100 \quad 1000$

2 year No. 1 Catawba........................... $30 \quad \$ 2.50 \quad \$ 20.00 \quad \$ 150.00$

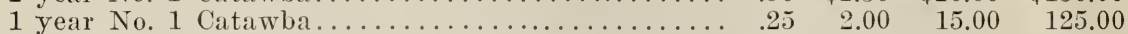

2 year No. 1 Agawam............................... $30 \quad 2.50 \quad 20.00 \quad 150.00$

1 year No. 1 Agawam............................. $25 \quad 2.00 \quad 15.00 \quad 125.00$

WHITE VARIETIES:

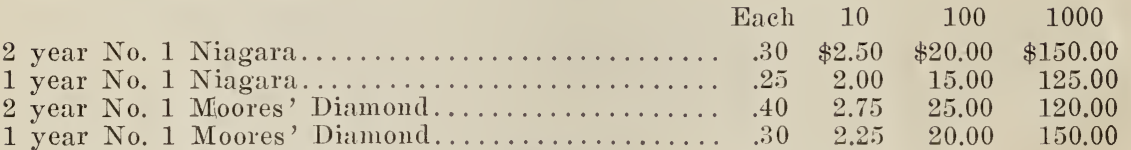

By Yarcel Post add 2c per plant extra.

\section{ASPARAGUS}

2 year Strong Plants, 10e each; 50e per dozen; $\$ 2.00$ per $100 ; \$ 10.00$ per 1000 .

1 year No. 1 , two-year size, 40 e per dozen; $\$ 1.50$ per $100 ; \$ 7.00$ per 1000 .

Varieties: Conovers, Palmetto, Barr's Mammoth, Col. White, and Washington.

By Parcel Post add 15e per 100 plants extra.

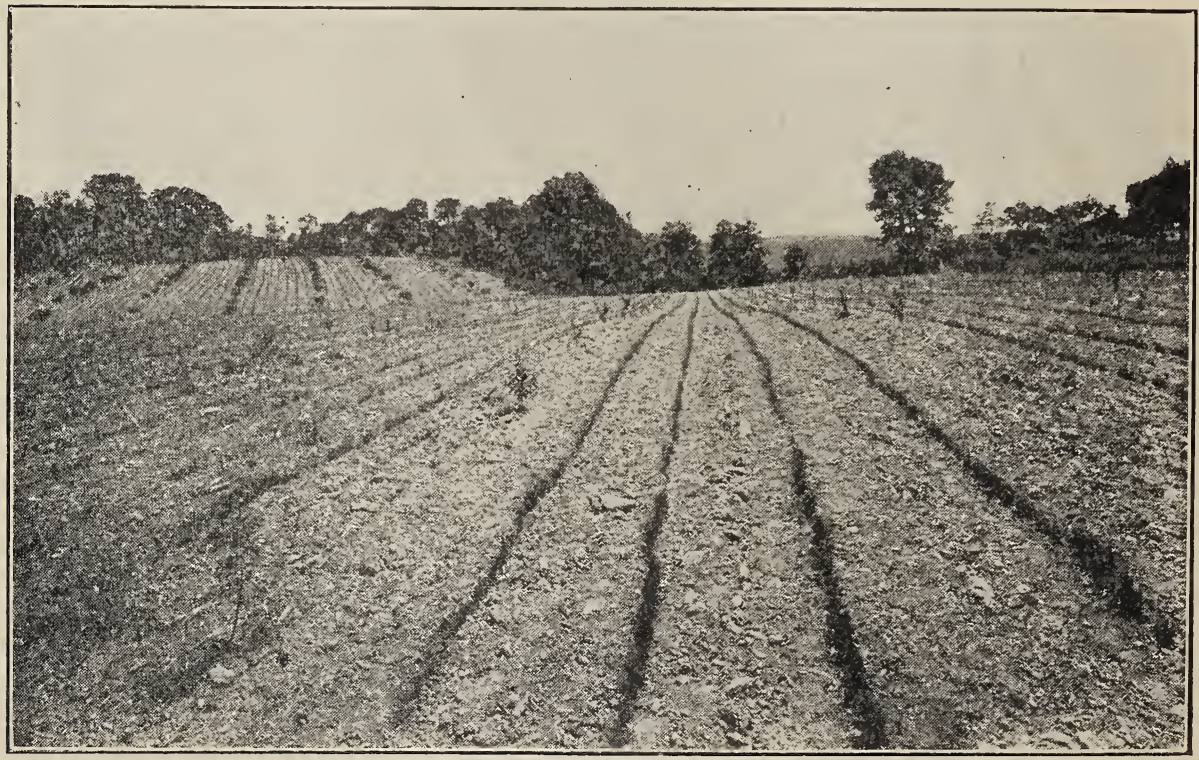

This shows two fields of our Young Orchards with 1 year Asparagus Plants consisting of about half million assorted varieties. Photographed July 10, 1924. 


\section{RHUBARB}

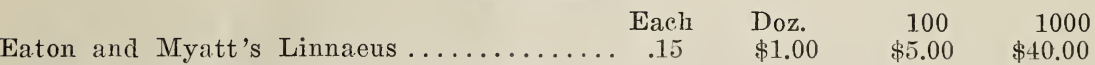

By Parcel Post add 1 1-2c per plant extra.

\section{STRAWBERRIES}

A.ny soil that will produce a good crop of corn is adapted to growing good Strawberries, but as they should be kept free from weeds, we believe it will pay to select land free from weed seed, even if it is not your richest land.

All plants of this class, tied 25 plants in a bunch; less the usual discount for cash with orders. Plants nicely packed on cars here. If by mail add 10c per 100 plants. No plants dug for shipment before October 1st.

\section{PRICES OF STRAWBERRY PLANTS}

\begin{tabular}{|c|c|c|c|c|}
\hline & 50 & 100 & 500 & 1000 \\
\hline Senator Dunlap & $\$ 1.00$ & $\$ 1.85$ & $\$ 5.00$ & $\$ 8.00$ \\
\hline Haverland $\ldots \ldots \ldots \ldots \ldots \ldots \ldots$ & 1.00 & 1.85 & 5.25 & 8.50 \\
\hline Steven's Late Champion ............. & 1.00 & 1.85 & 5.25 & 8.50 \\
\hline Chesapeake $\ldots \ldots \ldots \ldots \ldots \ldots \ldots \ldots \ldots$ & 1.25 & 2.00 & 6.00 & 10.00 \\
\hline Climax & 1.00 & 1.85 & 5.25 & 8.50 \\
\hline$\ldots \ldots \ldots \ldots \ldots$ & 1.00 & 1.85 & 5.00 & 8. \\
\hline Oak's Early . & 1.00 & 1.85 & 5.25 & 8.50 \\
\hline Klondike $\ldots \ldots \ldots \ldots \ldots \ldots \ldots \ldots$ & 1.00 & 1.85 & 5.25 & 8.5 \\
\hline Virginia $\quad \ldots \ldots \ldots \ldots \ldots \ldots \ldots \ldots$ & 1.00 & 1.85 & 5.25 & 8.5 \\
\hline Norwood $\ldots \ldots \ldots \ldots \ldots \ldots \ldots \ldots \ldots$ & 1.00 & 1.85 & 5.00 & 8.0 \\
\hline 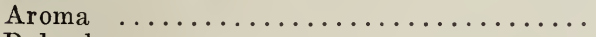 & 1.00 & 1.85 & 5.25 & 8.5 \\
\hline$\ldots \ldots \ldots \ldots \ldots \ldots \ldots$ & 1.25 & 2.00 & 5.50 & 9.0 \\
\hline e & 1.00 & 1.85 & 5.25 & \\
\hline$\ldots \ldots \ldots \ldots \ldots \ldots \ldots$ & 1.00 & 1.85 & 5.25 & 8.5 \\
\hline$\ldots \ldots \ldots \ldots \ldots \ldots \ldots \ldots \ldots$ & 1.00 & 1.85 & 5.25 & 8.5 \\
\hline
\end{tabular}

Other varieties priced on application. By Parcel Post add 1-2e plant extra.

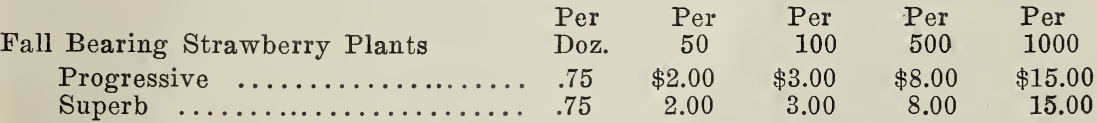

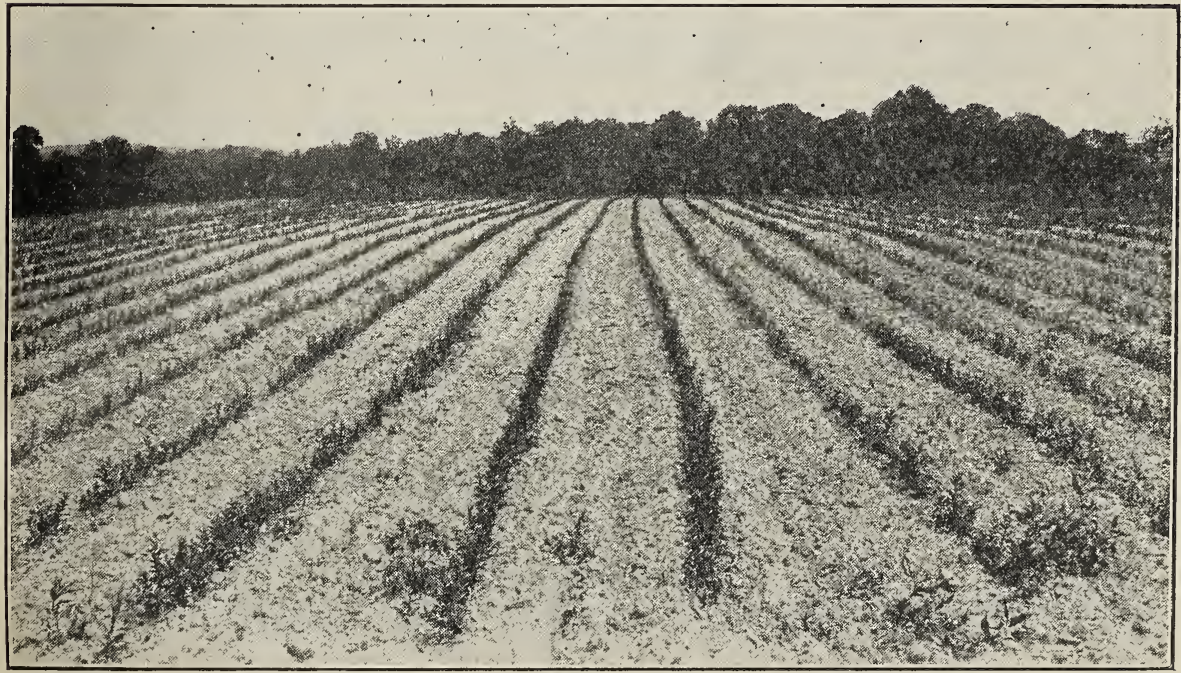

This shows a block of Cal. Privet coming one year, photographed July 10, 1924. Note excellent growth at this time. These are making up fine in 12 to 18, 18 to 24 and 24 to 36 inch grades. We have about 250,000 to offer. 


\section{PRIVET}

Price Per 10 Per 100 Per 1000

California 3 to 4 feet...................... \$1.75 $\$ 10.00 \quad \$ 80.00$

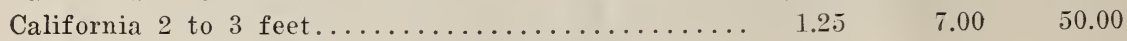

California $11-2$ to 2 feet...................... $1.00 \quad 4.00 \quad 35.00$

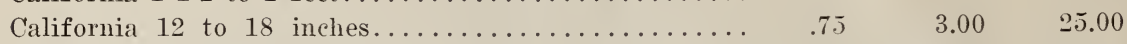

Armor River North 18 to 24 inches................... $1.25 \quad 7.00 \quad 60.00$

Armor River North 12 to 18 inches.............. $1.00 \quad 6.00 \quad 50.00$

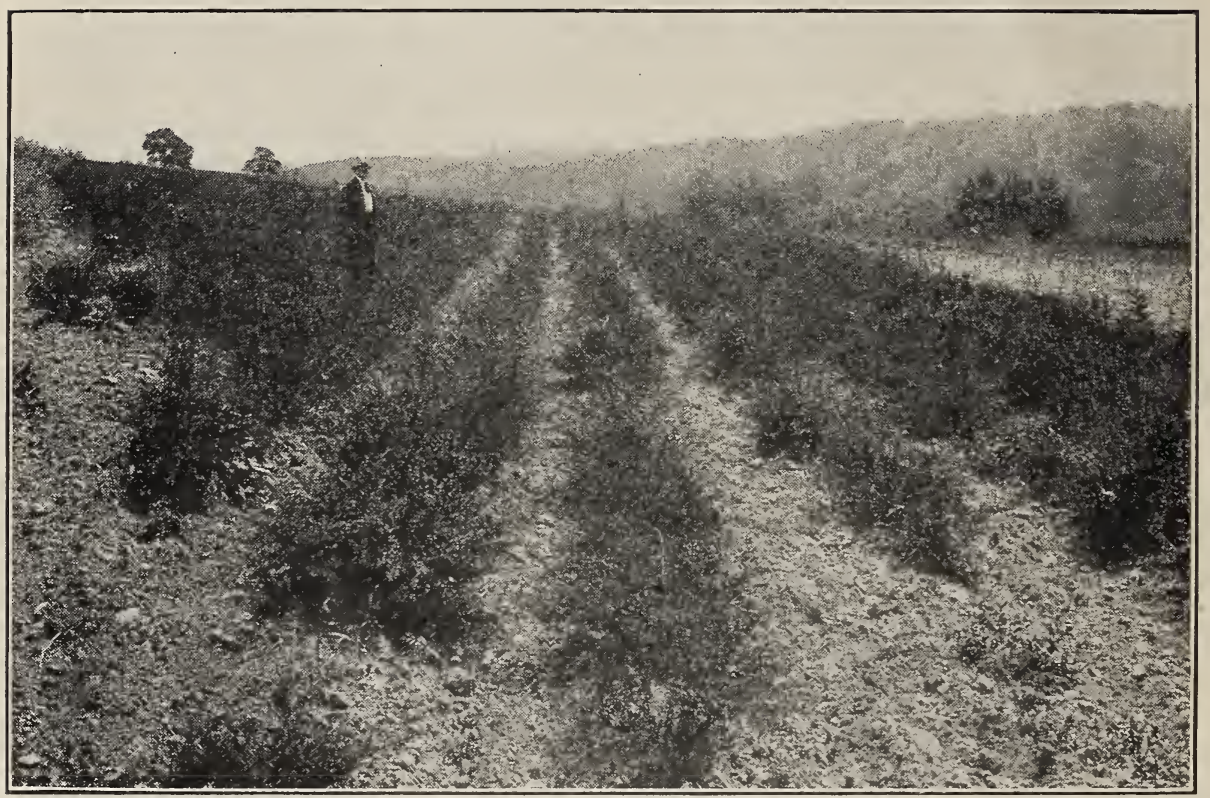

This is a very fine block of 3 year transplanted Barberry Thunbergi. Note nice bushy plants of 18 to 24 inch and 2 to 3 feet grades. J. E. Stoner standing in foreground. Photographed July 10, 1924.

\section{BARBERRY}

\begin{tabular}{|c|c|c|}
\hline & Per 10 & Per 100 \\
\hline Barberry Thunbergi 2 to 3 feet. & $\$ 4.50$ & $\$ 35.00$ \\
\hline Barberry Thunbergi 18 to 24 inches. & 3.50 & 20.00 \\
\hline Barberry Thunbergi 12 to 18 inches. & 3.00 & 16.00 \\
\hline Barberry Thunbergi 8 to 12 inches... & 2.00 & 10.00 \\
\hline
\end{tabular}

We have over 250,000 Privet, fine, bushy plants and these very low prices should interest you.

We Do Not Ship Privet or Barberry by Parcel Post.

NUT TREES

English Walnuts 3 to 4 feet ................... \$1.75

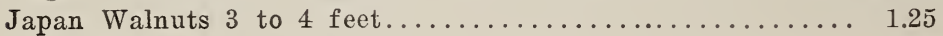

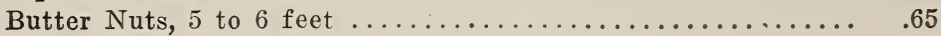

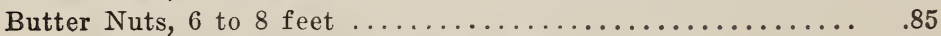

Grafted Chestnuts price on application. 


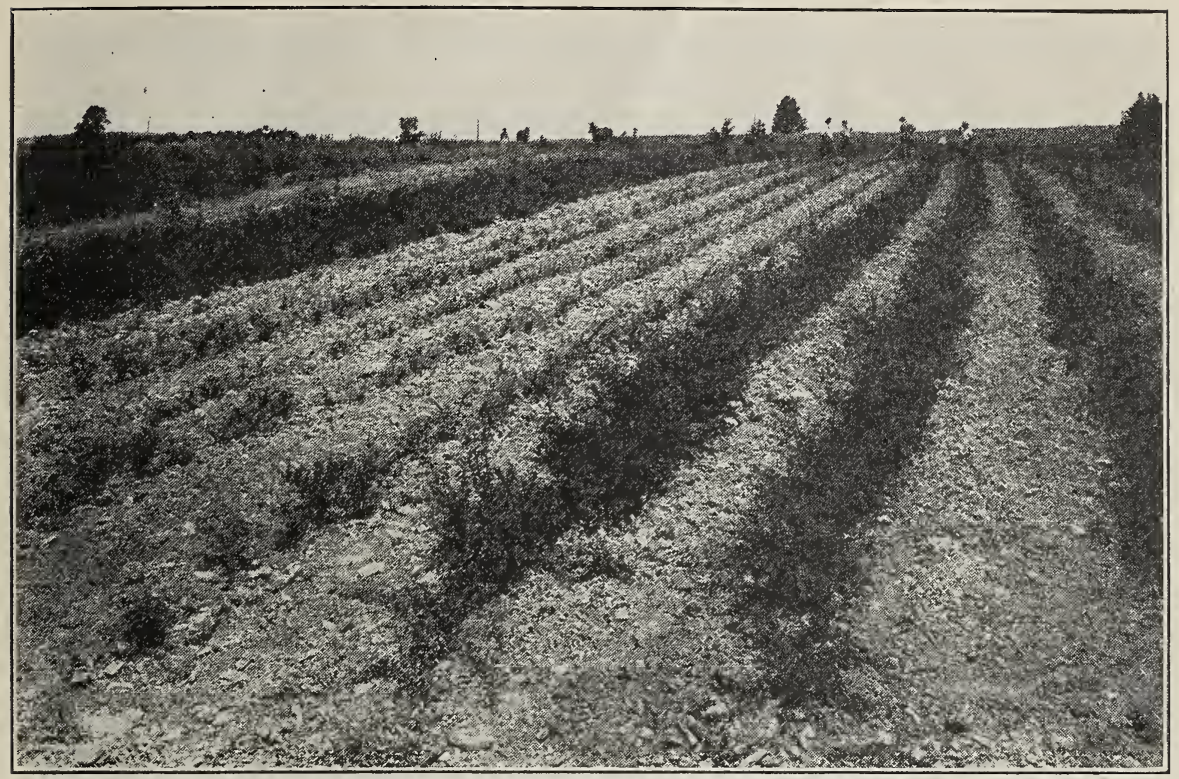

This shows one of our several blocks of one and two year transplanted Barberry Thumbergi, consisting of about 20,000 nice bushy plants. Photographed July $10,1924$.

\section{WFEPING TREES}

$\begin{array}{rr}\text { Each } & \text { Per } 10 \\ \$ 1.75 & \\ 1.50 & \\ 1.75 & \\ 2.50 & \\ 1.00 & \$ 8.50 \\ 2.00 & 17.50\end{array}$

\section{DECIDUOUS SHRUBS}

European Ash, 2 year heads ................. \$1.75

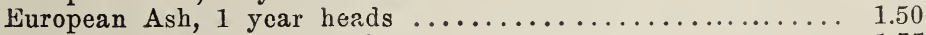

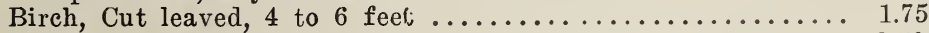

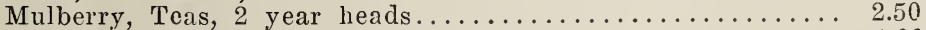

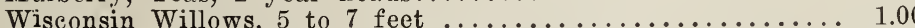

Umbrella Catalpa, 5 to 6 feet, select.............. 2.00

\begin{tabular}{|c|c|c|}
\hline & Each & Per 10 \\
\hline Althea, assorted, 2 to 3 feet. & $\$ .70$ & $\$ 6.50$ \\
\hline Almonds, double flowering, pink and white, 18 to 24 inches.. & .70 & 6.50 \\
\hline $\begin{array}{l}\text { btels, double flowering, American Crab } 2 \text { to } 3 \text { ft., the most } \\
\text { fragrant shrub that grows } \ldots \ldots \ldots \ldots \ldots \ldots \ldots \ldots \ldots \ldots \ldots\end{array}$ & & \\
\hline $\begin{array}{l}\text { fragrant shrub that grows } \ldots \ldots \ldots \ldots \ldots \ldots \ldots \ldots \ldots \ldots \ldots \ldots \\
\text { Butterfly Bush, No. } 1 \ldots \ldots \ldots \ldots \ldots \ldots \ldots \ldots \ldots \ldots \ldots \ldots \ldots \ldots\end{array}$ & 1.00 & 8.00 \\
\hline Butterfly Bush, No. 1....... & .75 & 6.50 \\
\hline 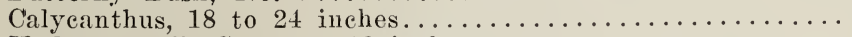 & .50 & 4.00 \\
\hline 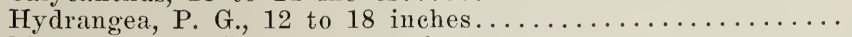 & .50 & 4.00 \\
\hline 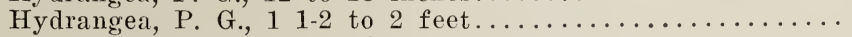 & .60 & 5.00 \\
\hline 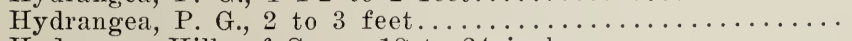 & .70 & 6.50 \\
\hline 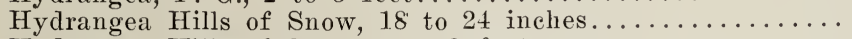 & .60 & 5.00 \\
\hline 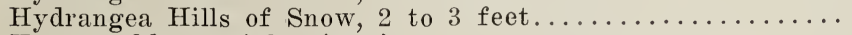 & .75 & 6.50 \\
\hline 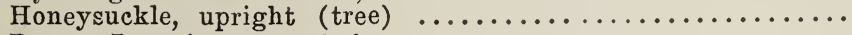 & .60 & 5.00 \\
\hline 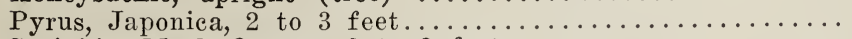 & .60 & 5.50 \\
\hline Syringia, Mock Orange, 2 to 3 feet........ & .50 & 4.00 \\
\hline 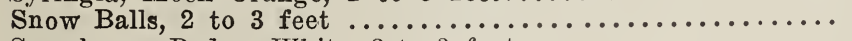 & .60 & 5.00 \\
\hline 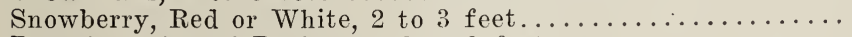 & .50 & 4.50 \\
\hline 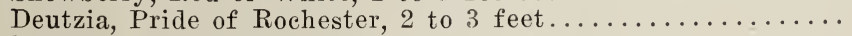 & .50 & 4 \\
\hline 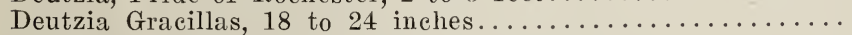 & .50 & 4.00 \\
\hline 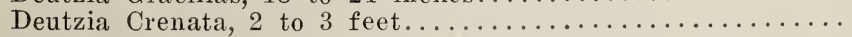 & .60 & 5.00 \\
\hline Forsythia (Golden Bell) 2 to 3 feet........ & .50 & 4.50 \\
\hline Fringe Purple, 3 to 4 feet.............. & 1.25 & 10.00 \\
\hline 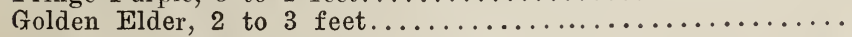 & .80 & 6.50 \\
\hline Kerris Japonica, Yellow, 2 to 3 fee & .60 & 5.00 \\
\hline lac, Pink, 2 to 3 feet. & .75 & 6.00 \\
\hline
\end{tabular}




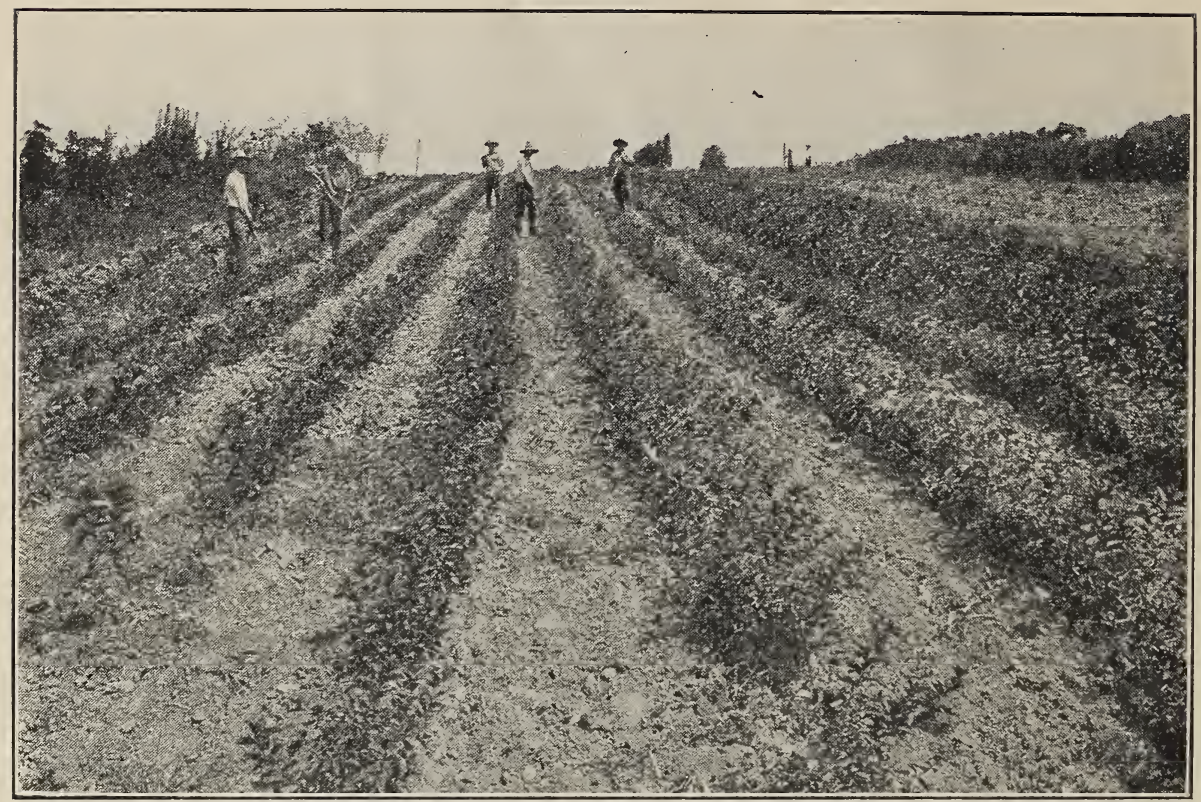

This is one of our blocks of Hydrangeas P. G. and Weigelias, which is making up fine in 12 to 18 inch and 18 to 24 inch grades. Pliotographed July 10, 1924.

\section{DECIDUOUS SHRUBS (Continued)}

Lilac, Persian, 2 to 3 feet..........................

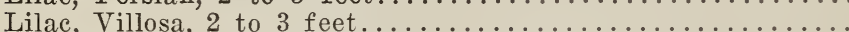

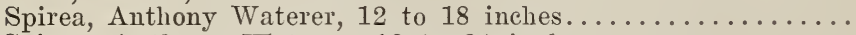

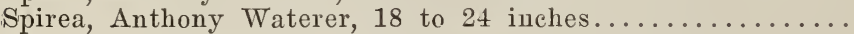

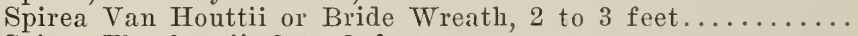

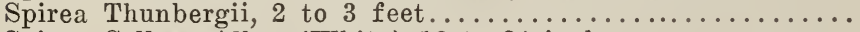

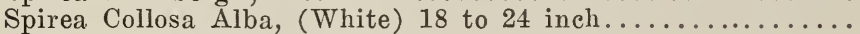

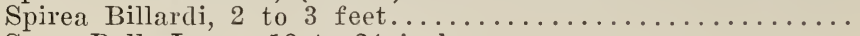

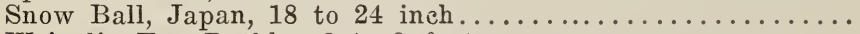

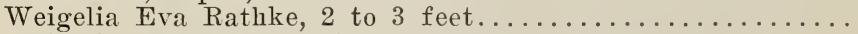

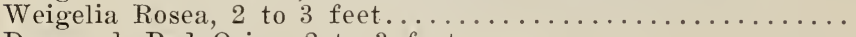

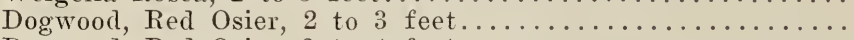

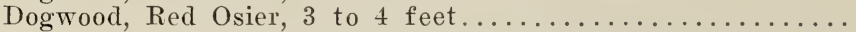

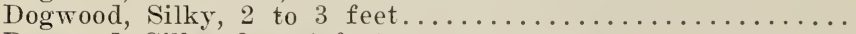

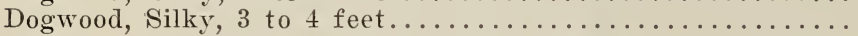

\section{IRIS}

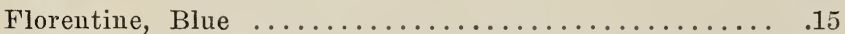

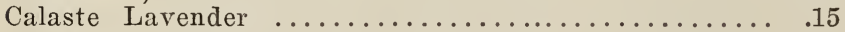

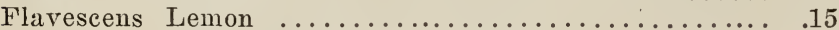

Gold Coin, Yellow splashed witl Wine............... .20

Leonidas Dark Blue ........................ ${ }_{20}$

Graccus, Yellow Petals ....................... .20

\section{CLIMBING SHRUBS}

Ampelopis, Virginia Creeper and Japan Ivy, strong plants...

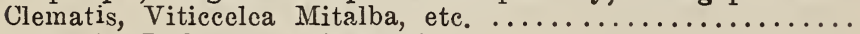

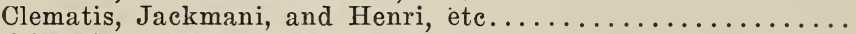

Wistaria, 2 year white and purple $\ldots \ldots \ldots \ldots \ldots \ldots \ldots \ldots . . .6$.

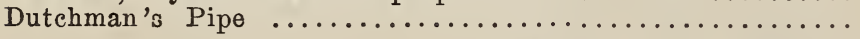

BULBS Each

Peonies, a good assortment, large flowering.........50

Yucca Filiamentosa, No. 1 (Adam's Needle)..........65

Dahlias, No. 1 red, whito and pink ................25

Foliage Cannas, roots or large growing foliage ..... 15
Each

1.00

1.00

.40

.50

.75

.75

.50

1.00

.60

.50

.40

.50

.40

.50

Each

\section{$\$ .60$}

.65

.90

.90

.90
Per 10

8.50

8.50

3.50

4.00

4.00

6.50

6.50

4.00

7.50

5.50

4.00

3.50

4.50

3.50

4.50

100

$\$ 10.00$

10.00

10.00

12.00

12.00

12.00

Per 10

$\$ 5.50$

6.00

8.50

8.50

8.50

100

$\$ 40.00$

10.00

8.00 


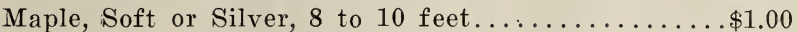

Maple, Soft or Silver, 6 to 8 feet........... 80

Maple, Norway, 10 to 12 feet............... 3.50

Maple, Norway or Sugar, 8 to 10 feet........... 2.50

Maple, Norway or Sugar, 6 to 8 feet............ 2.00

Maple, Japan, Red Leaf, 18 to 24 inches.......... 5.00

$\$ 8.00$

$\$ 70.00$

6.50

60.00

30.00

250.00

25.00

200.00

Maple, Japan, Red Leaf, 2 feet................ 6.00

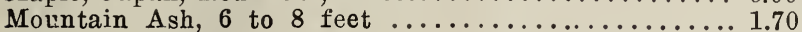

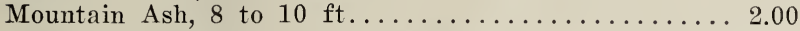

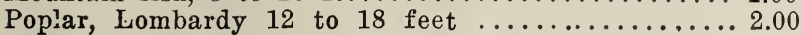

Poplar, Lombardy, 10 to 12 feet.............. 1.50

Poplar, Lombardy 8 to 10 feet................ 1.00

Linden, European and Anerican, 12 to 16 feet...... 5.00

Linden, European and American, 8 to 10 feet ........2.00

Poplar, Tulip and Silver, 8 to 10 feet............ 1.50

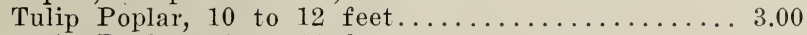

Tulip Poplar, 12 to 14 feet................. 4.00

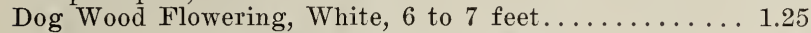

Dog Wood Flowering, White, 5 to 6 feet........... 1.00

15.00

135.00

Dog Wood Flowering, White, 4 to 5 feet........... .75

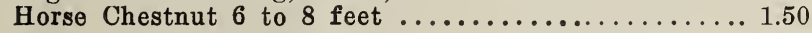

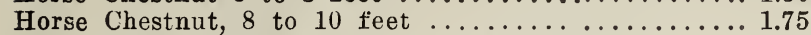

Horse Chestnut, 10 to 12 feet, $23-4$ in. cal. ....... 4.50

Magnolia, 3 to 4 feet, assorted............. 4.50

Our Shade Trees cannot be beaten.

15.00

17.00

18.00

12.50

9.00

18.00

12.00

25.00

35.00

10.00

8.00

6.00

12.00

15.00

40.00

40.00

Prices on other sorts and sizes given on application.

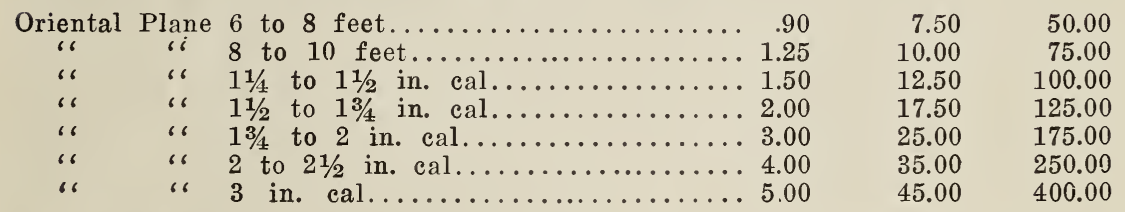

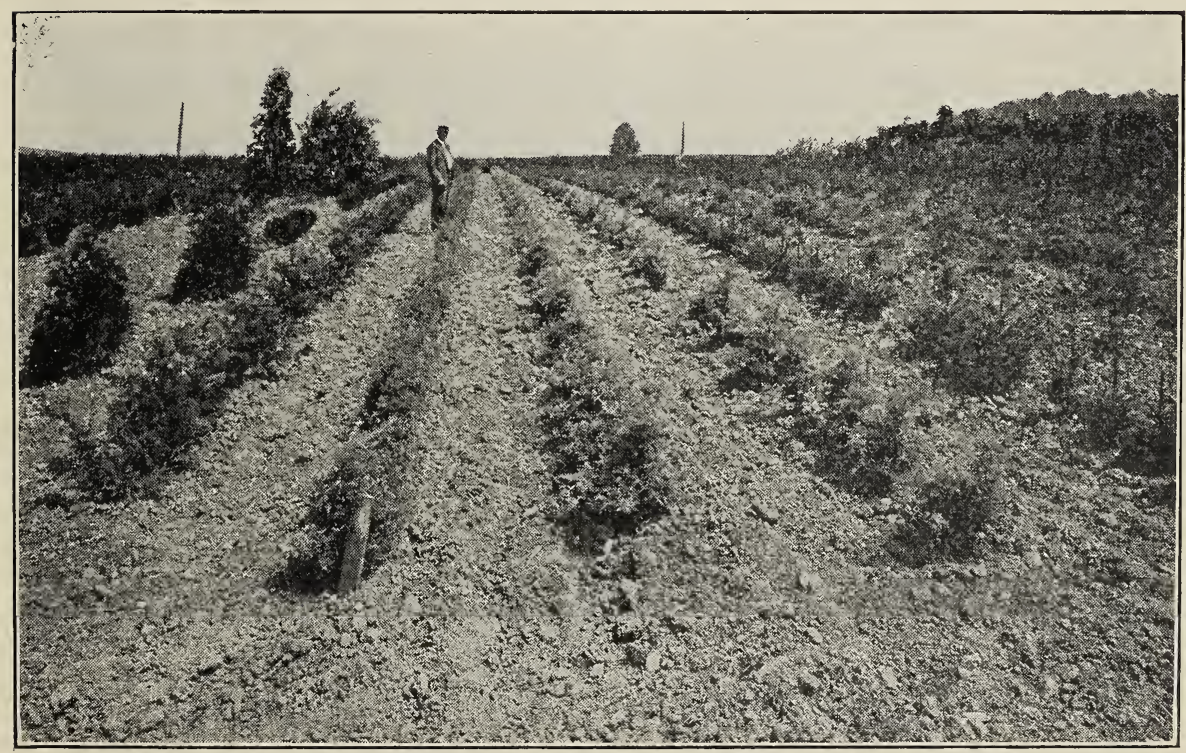

One block of our Evergreens showing in center Retinaspora, Squarrosa Veitchii (Silver). To the right Norway Spruce, Ete., and to the left Boxwood, Etc. J. E. Stoner standing in the foreground. Photographed July 10, 1924. 


\section{EVERGREENS:}

Each

10

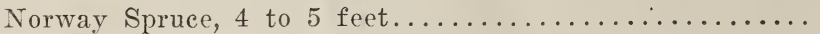

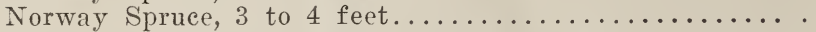

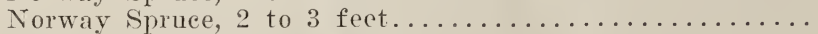

Norway Spruce, 18 to 24 inch...................

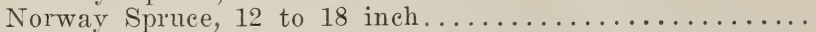

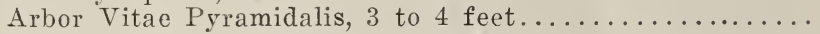

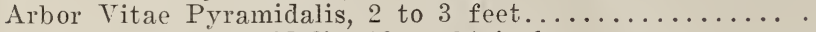

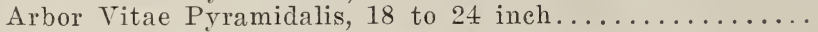

Arbor Titae Pyramidalis, 12 to 18 inch..............

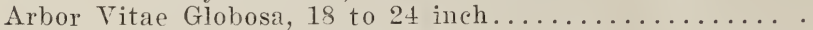

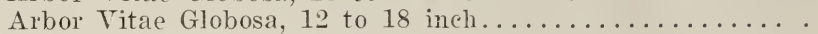

Arbor Vitae Hoveyii, 18 to 24 inch................

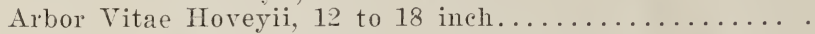

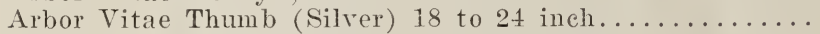

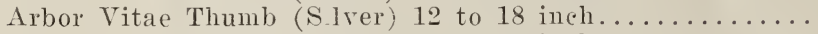

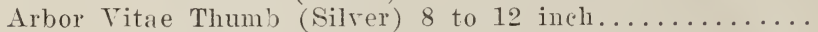

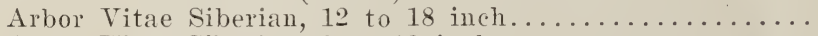

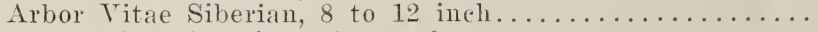

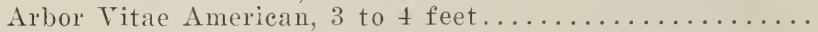

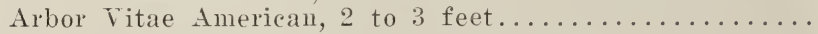

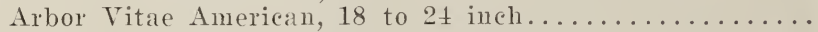

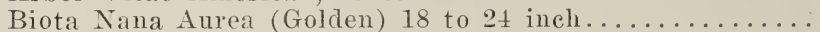

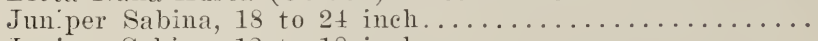

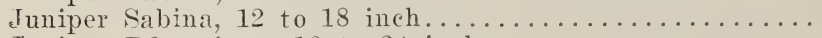

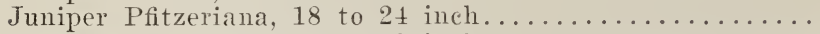

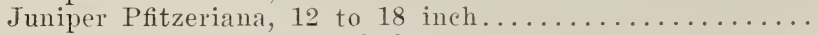

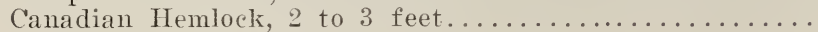

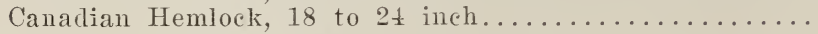

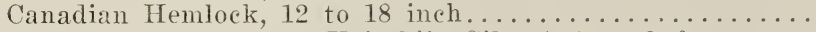

Retinaspora Squarrosa Teitchii (Silver) 2 to 3 feet.....

Retinaspora Squarrosa Teitchii (Silver) 18 to 24 inch....

Retimaspora Squarrosa Teitchii (Silver) 12 to 18 inch ....

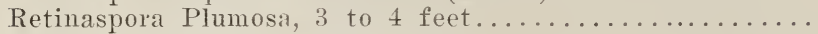

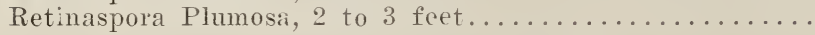

$\$ 4.00$

3.00

1.50

1.2 .5

.75

3.00

2.50

2.00

1.00

2.75

2.00

2.75

2.00

2.75

2.00

1.50

2.00

1.50

2.25

1.75

1.35

3.00

3.00

2.00

2.75

1.85

2.50

2.00

1.50

3.50

2.75

1.8 .5

3.25

2.75

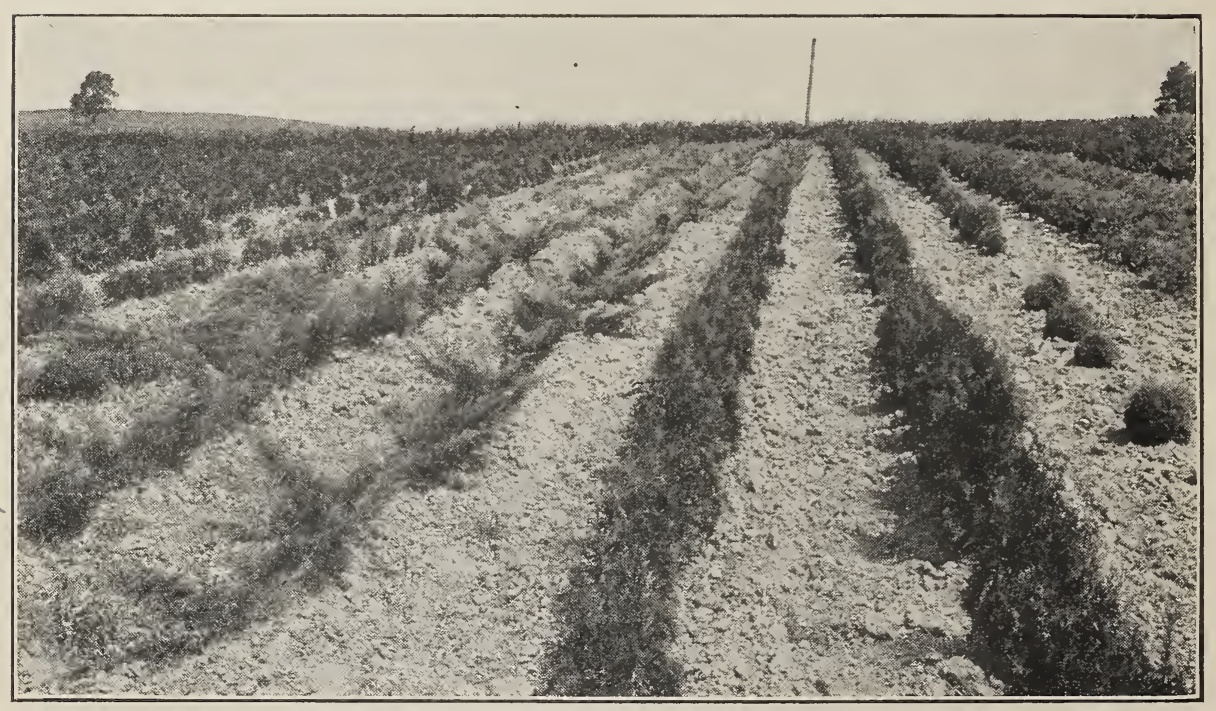

This is a block of Evergreens showing Arbor Vitae, Pvramidalis and Junipers Sabina, Etc. Also note other assorted varieties of Evergreens to right and left, all of which are nice popular size for immediate effect. Photographed July 10, 1924.

We call your attention to our Evergreens and low prices. August 25th to September 25th is the proper time to transplant all evergreens. We dig all Evergreen with a good ball of earth and then burlap them. 


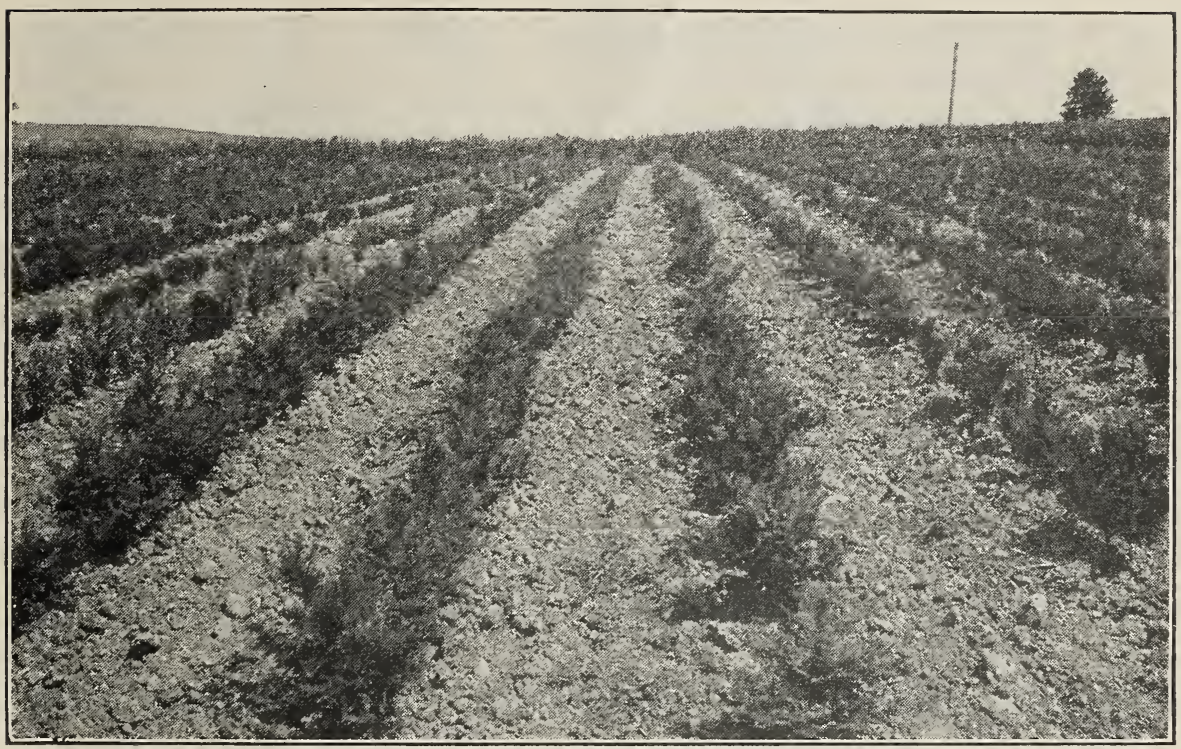

A block of our Evergreens showing Retinaspora Plumosa and Aurea (Golden) Note nice size of 18 to 24 inch and 2 to $3 \mathrm{ft}$. grades. Photographed July 10, 1924.

\section{EVERGREENS (Continued)}

Retinaspora Plumosa, 18 to 24 inch.

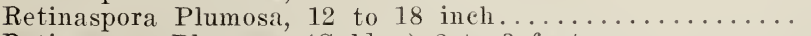

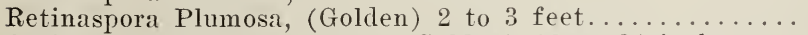

Retinaspora Plumosa Aurea, (Golden) 18 to 24 inch......

Retinaspora Plumosa Aurea, (Golden) 12 to 18 inch.......

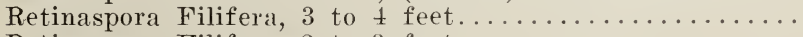

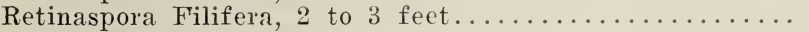

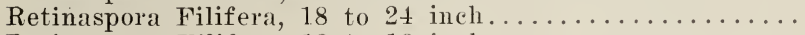

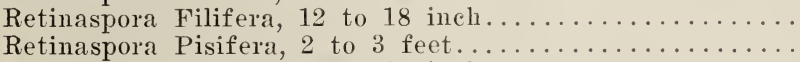

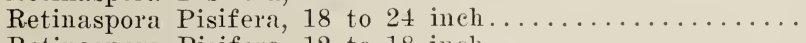

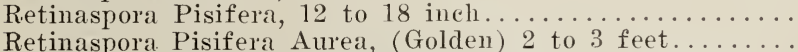

Retinaspora Pisifera Aurea, (Golden) 18 to 24 inch...... Retinaspora Pisifera Aurea, (Golden) 12 to 18 inch......

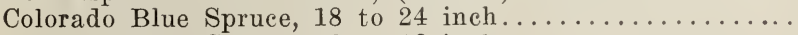

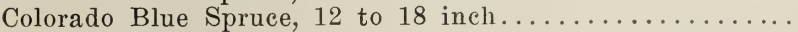

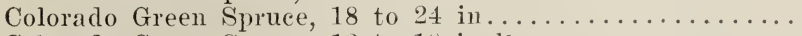

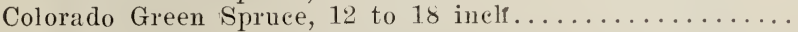

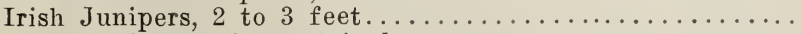

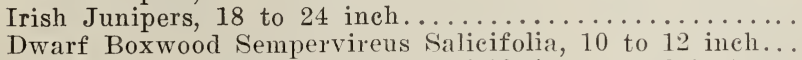
Dwarf Boxwood Sempervireus Salicifolia, $\delta$ to 10 inch.... Dwarf Boxwood Sempervireus Suffruticosa, 10 to 12 inch.. Drvarf Boxwood Sempervireus Suffruticosa, 8 to 10 inch..

$\begin{array}{rr}\text { Each } & \text { Per } 10 \\ \$ 2.25 & \$ 20.00 \\ 1.85 & 17.50 \\ 3.00 & 27.50 \\ 2.50 & 22.50 \\ 2.00 & 18.50 \\ 3.00 & 27.50 \\ 2.50 & 22.50 \\ 2.00 & 17.50 \\ 1.50 & 12.50 \\ 2.75 & 25.00 \\ 2.25 & 20.00 \\ 1.85 & 17.50 \\ 3.00 & 27.50 \\ 2.50 & 22.50 \\ 2.00 & 18.50 \\ 3.50 & 30.00 \\ 2.50 & 20.00 \\ 1.50 & 12.50 \\ 1.25 & 10.00 \\ 2.00 & 18.00 \\ 1.50 & 12.25 \\ 1.00 & 9.00 \\ .75 & 7.00 \\ 1.00 & 9.00 \\ .75 & 7.00\end{array}$

Standard Boxwood Sempervireus Arborescebs

(Tree Box) 12 to 18 inch, Bushy.............. \$1.25

(Tree Box) 18 to $2+$ inch, Bushy................ 2.00

(Tree Box) 24 to 30 inch, Bushy............... 2.75 25.00

Boxwood will help greatly to beautify your home. We have a good supply of nice bushy plants. Our Boxwoods are dug with ball and burlap. August 25th to September 31 st is a good time to transplant Boxwood. 


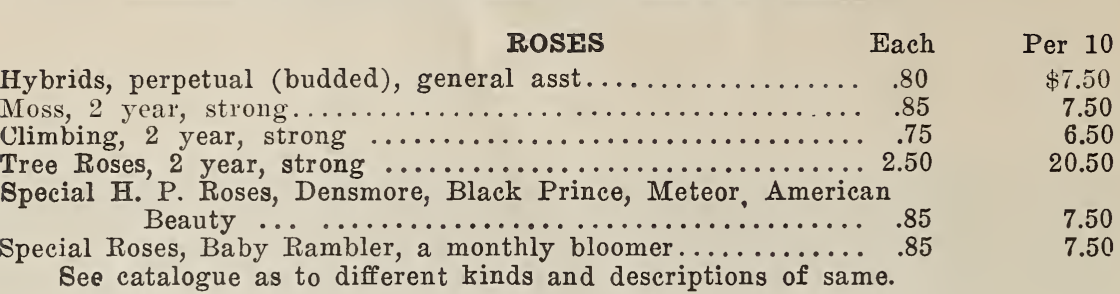

\section{NUMBER OF TREES TO AN ACRE}

30 feet apart each way 50

25 feet apart each way 75

20 feet apart each way 110

18 feet apart each way 135

15 feet apart each way 205

11 feet apart each way 300
10 feet apart each way 345

8 feet apart each way 684

6 feet apart each way 1,210

5 feet apart each way 1,742

4 feet apart each way 2,723

3 feet apart each way 4,840

RULE-Multiply the distance in feet between the rows by the distance the plants are apart in the rows, and the product will be the number of square feet for each plant or lill, which divided into the number of feet in an acre, 43,560, will give the number of srees in an acre.

Standard Apples

Standard Pears and strong growing Cherries

Standard Plums, Apricots, Peaches, Nectarines

Dwart Pears

Grapes

Strawberries, for field culture

Strawberries, for garden culture

Raspberries
30 feet each way

20 feet each way

14 to 18 feet each way

10 to 12 feet each way rows 8 to 10 feet apart, 7 to 8 feet in rows

1 by 4 feet apart

1 to 2 feet apart rows 4 to 6 feet apart, 2 to 3 feet in rows

SHIPMENTS BY PARCEL POST cannot be larger than 72 inches including the length and circumference of the package. Weight must not exceed 70 pounds within the First and Second Zones-that is within a radius of 150 miles of the shipping point; elsewhere, not more than 20 pounds can be sent by Parcel Post.

Parcel Post. Trees not larger in size than 2 to 3 feet can be sent by parcol post prepaid, at 3 cts. per tree extra.

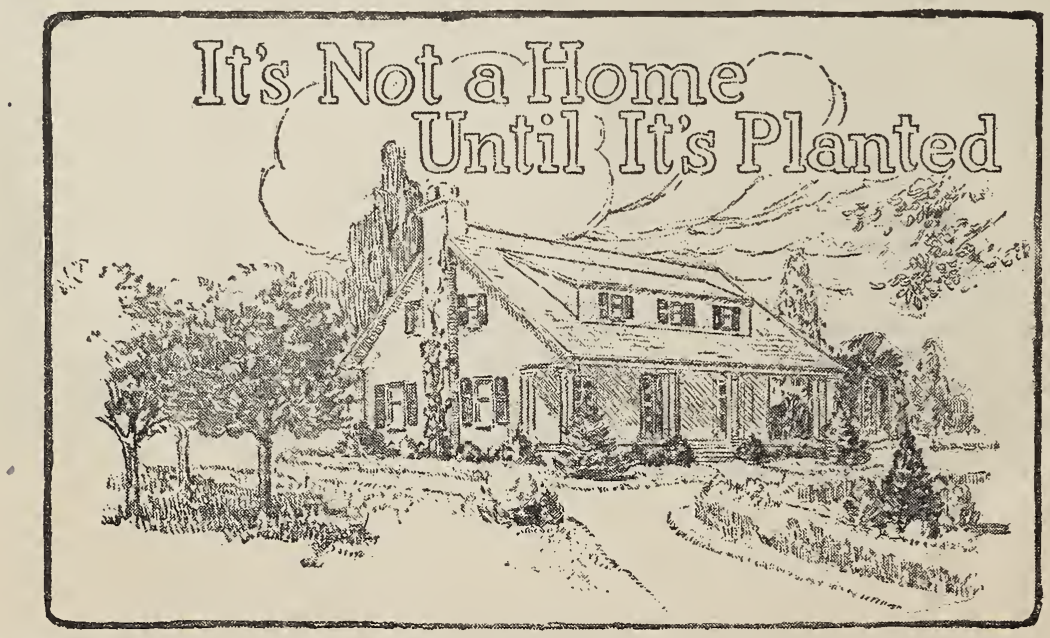

We sell only trees we grow. This is for your protection as well as our own. Our trees always prove to be just what they are sold for. 


\section{NOW IS THE PROPER TIME TO PLANT AN ORCHARD}

WHY?

Because there are less Peach and Apple trees in the United States today than there were in 1910.

The following figures were copied from a bulletin issued by the

Department of Commerce, Bureau of The Census, Washington, $D$. C. dated June 27, 1921.

APPLES :

Trees of bearing age-

1920 .

$115,265,029$

1910

$151,322,840$

Decrease

$36,057,811$

Per cent of decrease

23.8

Trees not of bearing age-

1920

$36,171,604$

1910

$65,791,848$

Decrease.

$29,620,244$

Per cent of decrease

45.0

PEACHES :

Trees of bearing age-

1920.

$65,654,921$

$94,506,657$

Decrease

$28,851,736$

Per cent of decrease............................................... $\quad 30.5$

Trees not of bearing age-

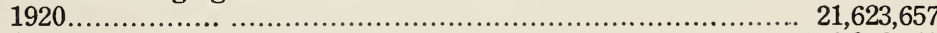

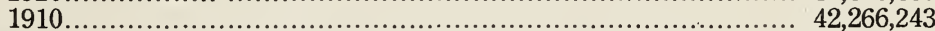

Decrease

$20,642,586$

Per cent of decrease.

48.8

Another reason why every fruit grower should increase his planting is that our prices on peach and apple trees have been reduced more than 50 per cent.

On the other hand, prices of fruit this season are high---much above the prewar basis.

The Westminster Nursery,

Westminster, Md. 



\section{Advantages of Fall Delivery of Nursery Stock}

\section{What a Leading Horticultural Journal Says:}

In our own experience the best success has resulted in transplanting trees and shrubs in the Fall, in properly prepared soil, and giving Winter protection by mulching with litter of leaves. Such subjects usually start to grow promptly in Spring, and have already made a fine growth before Spring-planted trees have started. We do not hesitate to advise Fall planting in climates not more severe than our own.-Dick's Magazine.

Some of the reasons why experienced planters like to buy for Fall delivery are as follows :

1st.-All kinds of stock, except Evergreens and Peaches, in cold sections do well when planted in the Fall. warm.

2d.-The ground is in much better condition in the Fall, being mellow and

3d. Before Spring, stock which has been planted in the Fall gets well established in its new position and so gets an early start, and makes a large growth the first season, while stock planted in the Spring loses considerable valuable time in getting established in its new quarters.

4th.- Stock is completely dormant in the fall, and therefore can be easily and safely handled, while in the Spring the weather often comes off warm so early as to start the buds seriously before the customer can receive and plant his stock.

5th.- Stock planted in the Fall gets the benefit of the late Fall and early Spring rains, which is of vital importance; on the contrary, stock set in the Spring frequently has to worry through a dry Summer, which it is utterly unprepared to do after having been so newly transplanted. The result is that its growth is stunted.

6th.-Fruit is produced the first Summer when Grape Vines, Currants, Raspberries, Blackberries, Gooseberries, ete., are planted in the Fall.

7th.-Rose Bushes and Shrubs set out in the Fall bloom profusely the first Summer.

8th.-Farmers and others have more time to attend to stock in the Fall, and ean therefore give it better eare, which is very important. Much stock is lost by farmers when planted in the Spring because their farm work presses so then that the stock is neglected.

9th.-Stock delivered in the Fall arrives in better order, because Nurserymen have more time in which to handle it; but in the Spring it comes off warm so suddenly that it is time to plant before the stock can possibly be dug, packed and delivered to all the thousands of customers seattered over the country.

10th. The railroads are crowded and overworked in the Spring so that it is impossible to get stock through as rapidly as in the Fall, and the result is more or less serious delay.

11th.-Many extensive and experienced planters, like the late horticultural writer, E. P. Roe, always prefer to plant in the Fall. (See extract at top of this cireular from Vick's Magazine.)

12th.-Even if the customer is not able to plant until Spring, or the elimate is too cold, it is wise to have the stock delivered in the fall, so that he can trench it in carefully, and seize the earliest moment in the Spring to set it out, instead of losing valuable time in the Spring waiting for the overworked nurserymen and railroads to get the stock around.

\section{HOW TO " HEEL IN", OVER WINTER}

There is a popular impression that trees dug in the Fall and heeled in over winter are worthless. If the heeling is well done it is perfectly safe. Peach and some other young trees, if left standing during their first Winter, are frequently killed or injured by frost, while if dug in the Fall and treated as below deseribed, they come through bright and uninjured.

To insure success select a dry spot where no water will stand during Winter, having no grass near to invite mice. Dig a trench deep enough to admit one layer of roots, and sloping enough to permit the trees to lie at an angle of not more than 30 degrees with the ground. Having placed one layer of roots in this trench, cover them with mellow earth extending well up on the bodies, and see that this is firmly packed. Then add another layer of trees, over-lapping the first, continuing as at first until all are heeled in. As soon as this is done, cover the tops so well with evergreen boughs that they will be thoroughly protected from winds. Roses and other small stock may be wholly covered with earth. 



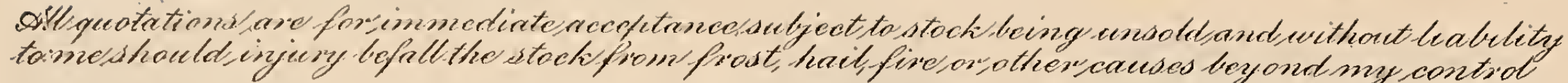

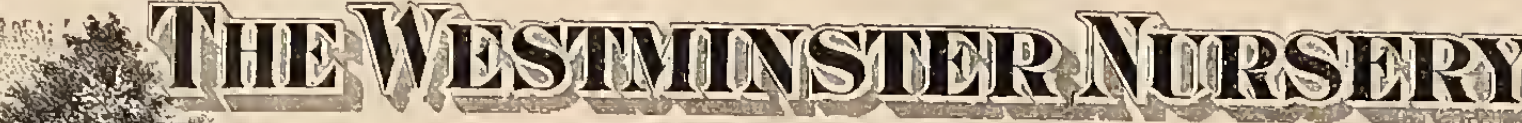

J. E. STONER, PHOPTIETOR

WHOLESALE ANDD RIEUAII

$c$

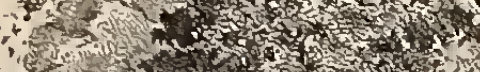

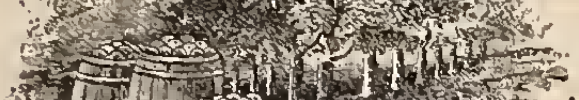

PEACH, APPLE, PEAR, CHERRY AND PLUM TREES

ASPARAGUS AND BERRY PLANTS, CALIFORNIA PRIVETS

AND BARBERRY THUNBERGI, SHRUBBERY AND ORNAMENTALS

ALL TREES FUMIGATED

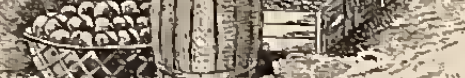

LONG DISTANCE C \& P. TELEPHONE ZZZ

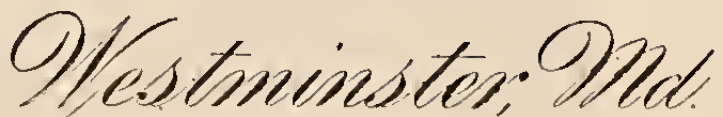

\section{Dear Sir:-}

Nearly everyone is more or less interested in fruit growing, or beautifying his home whether it be in a commercial way, or merely for home growing. Therefore we take pleasure in presenting you our Planter's Price List. May we not ask you to look it over carefully? There is much in it that may appeal to you and which is worthy of the special attention of anyone wishing to secure nursery stock of the BEST quality. We call your attention to our reduced prices on peach and apple trees, asparagus and rhubarb plants,

California Privets, Barberry, Evergreens, etc.

Our prices should attract your attention and bring us your orders, which we assure you will have our personal attention.

We have spent a lifetime in growing and handling Nursery Stocir and our methods are modern and facilities unsurpassed.

Our aim in the Nursery business is to produce good trees, true to name and to grade them according to the highest standard. The leading planters of the west, north, east and south will vouch for the high quality of our trees and our prompt, courteous efficient service.

You can save money by dealing with us. You can get your trees on time and get them true to name and strictly up to grade. Just write us to see how careful and prompt we are about every detail of our business. Ask for special prices on large orders.

Results are what you want; and in order to get the best results you must first of all have the BEST trees.

Our prices are not raised to cover losses from bad accounts--we have none. We sell for cash and our customers save all middlemen's profits.

CATALOG--Will be mailed on request. We allow 5\% discount when all cash with orders, at prices named in Price List that are received oefore Oct. Ist, and $3 \%$ thereafter for Fall Shipment; and for Spring Shipment 5\% discount for all cash with orders before March 1st, and 3\% thereafter.

We will ship large orders C. 0. D. Provided 25 per cent of total amount of your order is sent in cash. If order is sent by express you pay the balance to your express agent upon delivery of goods. If the order goes by freight you pay the balance at your bank, where you will also find the bill of lading which you present to your freight agent who will deliver shipment to you.

We trust to receive your early orders, which will have our careful attention, and if there is anything about our Price List you do not understand kindly advise us and we will explain.

Awaiting your commands, and thanking you in advance for a reply, I remain, Yours very truly,

Everything being equal we will meet the prices of any reliable competitor.

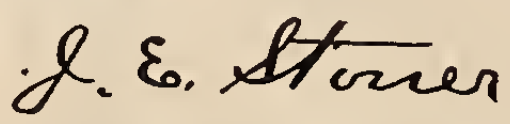

P. S. Should you be in the market for a quantity of stock and will mail us a list stating the number of each variety and size of trees and plants wanted we will name special letter prices on same, 

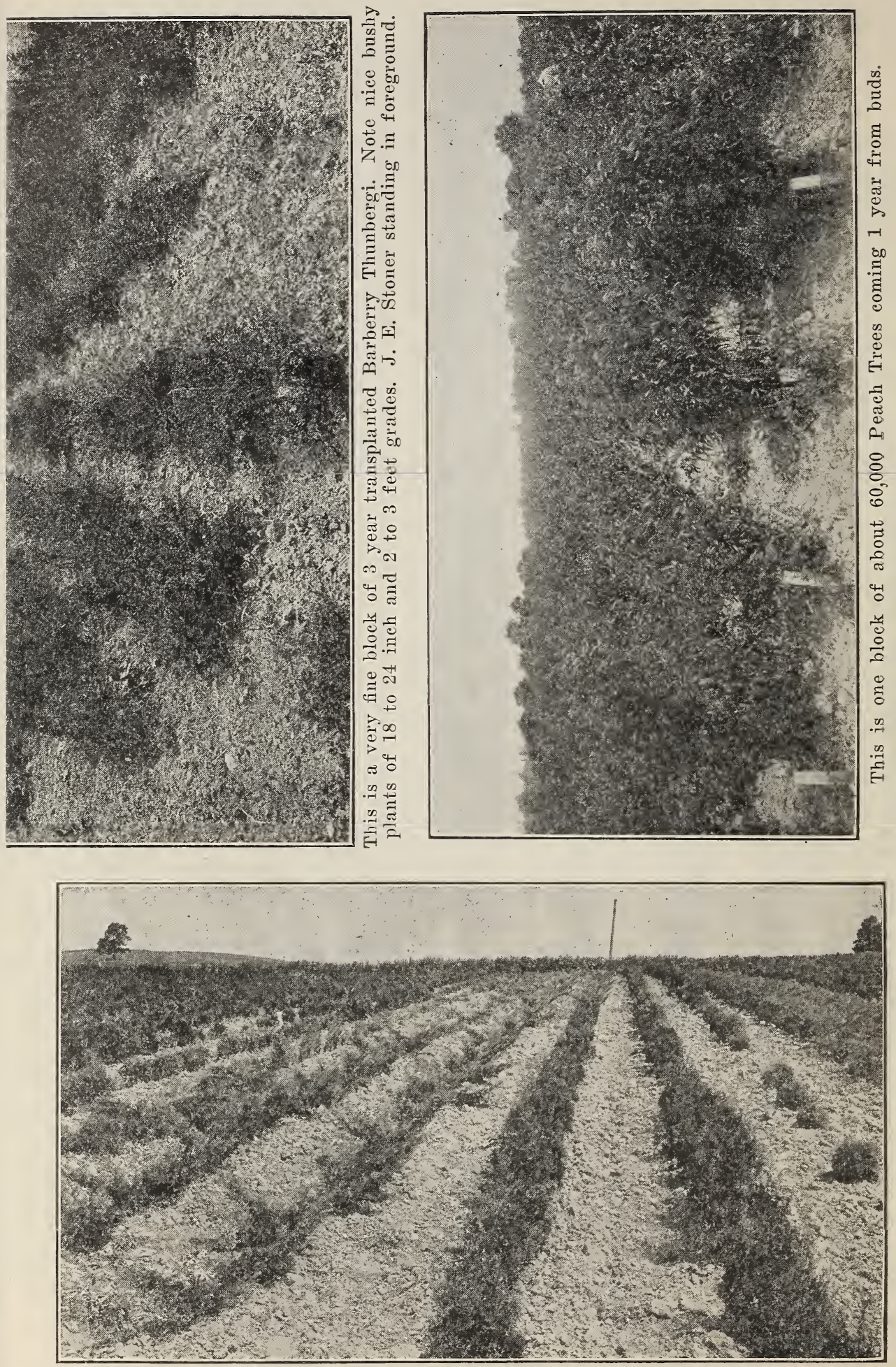

This is a block of Evergreens showing Arbor Vitae, Pyramidalis and Junipers Sabina. 


\section{SPRAYERS AND SPRAYING SOLUTION}

We sell Sprayers and instruct kind of spraying and teach how and when to spray. We offer Lime Sulphur Solution, Arsenate of Lead, Soluble Oil, etc., at low prices.

\section{SPRAYING CALENDAR}

For information about insects, pests and plant diseases, write to the Experiment Etation in your state.

Oyster Shell Bark Louse-Attacks apple mainly; use sulphur-lime spray when the leaves are off the trees. If this spraying is ineffectively done, apply kerosene emalsinn or whale oil soap when eggs hatch.

San Jose Scaie-Attacks nearly all deciduous trees; use sulphur-lime for spray in the spring before the buds burst.

Red Spider-Attacks fruit trees and bushes; spray with sulphur-lime in the spring before the buds burst. This will kill the winter eggs. If the mite appears in summer, use kerosene emulsion, best adding one ounce of sulphur to the kerosene $f \cdots$ each gallon of spray, or use sulphur-lime $1 \frac{1}{1 / 2}$.

Green Aphis-Attacks pear, peach, plum, prune trees, etc.; spray with sulphurlime in the spring before the buds burst. If the insects appear, spray with tobacco into the curling leaves.

Woolly Aphis-Attacks apple trees; use sulphur-lime, kerosene emulsion or tobacco for spraying, just before the buds burst. Spray with force. For summer t-eatment use kerosene or tobacco.

Slug-Attacks pear and apple trees; use arsenate of lead for spray when in. sects uppear, or dust with lime, road dust or ashes.

St:ab-Attacks apple and pear trees; spray with sulphur-lime $11 / 2$ just before the blossoms open and again while the last blossoms are falling.

\section{BORDEAUX}

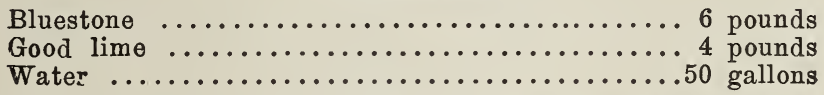

Dissolve the bluestone by suspending it in a sack in 25 gallons of water in a barrel. Slake the lime in another vessel, adding a little water slowly, and dilute to 25 gallons. Mix the two thoroughly. Even the best Bordeaux may scorch in rainy weather For double strength Bordeaux use twice as much bluestone and lime

\section{WHALE OIL AND QUASSIA}

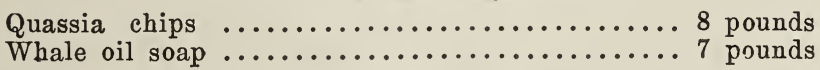

Soak chips twelve hours in eight gallons of water. Dissolve the soap in boiring water. Strain the quassia extract to remove the chips and add the soap solution. Stir thoroughly and dilute to make 100 gallons. This solution is used almost exclusively for the hop aphis. It is almost as effective without the quassia against other species of aphis. Soap powder or laundry soap may be used in tne same proportion without the quassia for most aphides.

\section{SULPHUR-IIME}

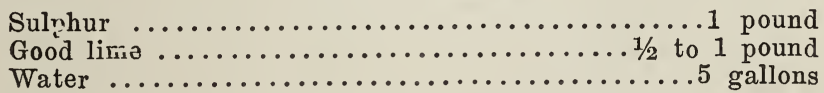

First slake the lime in the cooking vat. When slaked add the sulphur and about one-fifth of the water, so that the mixture will boil easily. Keep it well stirred. Boil until the sulphur is completely dissorved, which should take less than one hour. Then add the rest of the water and the mixture is ready to spray. Use only the clear liquid. It should be poured into the spraying tank through a strainer. It may be used hot or cold. There are several ready-made sulphurlime washes which give good results when properly diluted. When mixed 1 part to 10 parts of water they usually correspond in strength to the formula above. 
ARSENATE OF IEAD

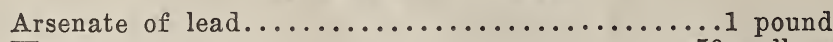

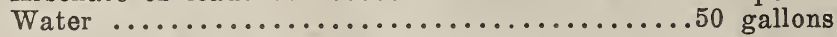

It is unnecessary to use it stronger. It is more reliable than Paris Green. It is especially useful where there is much rain. It sticks well and does not scorch. Mix well first with a small amount of water.

\section{KEROSENE EMULSION}

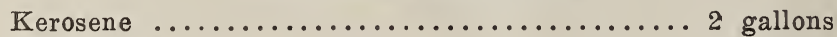

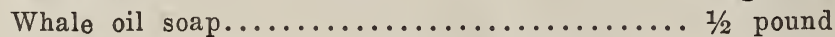

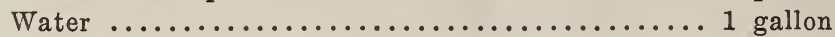

Dissolve the soap in the water by boiling and add the suds boiling hot to the kerosene, away from the fire. The mixture is then to be agitated violently, preferably by pumping it back into itself with a force pump. After four or five minutes the mizture suddenly becomes creamy in consistence. If well made the cream will stand for a long time without free oil rising to the surface. Use one gallon of the emulsion to 12 gallons of water in spraying. One quart of soft soap or one pound of laundry soap may be used instead of the whale oil soap.

If Frozen when Received bury the package unopened in well drained ground or place it in a cool cellar, so that it will thaw out slowly and gradually without being exposed to the air.

If they should appear Dry or Shriveled When Received, through delay in transit, or from any cause, take them from the package and plunge into a tub of water or bury the roots in the ground in an inclined position, so as to cover onehalf or more of the tops with earth, and then thoroughly soak with water, and then let remain for twenty-four hours or more, until they regain their fresh, plump appearance, when they may be planted.

Our Guarantee-While we exercise the greatest care to have every tree and plant true to name and are ready, on proper proof, to replace anything sent by us that proves untrue to label, free of charge, it is understood and agreed between purchaser and ourselves that we are not to be held liable for any greater sum than that paid us for said trees that may prove untrue.

Time of Shipment-To the Fall trade, we will commence to ship trees as soon as the leaves are off and the wood ripe, and plants about the 1st of October and continue as long as the weather is mild. For the Spring trade, in most seasons; we can fill orders as early as February for the South, as late as May for the Northern customers. Always name date when you want stock shipped but have it reach you before you need it. We endeavor to handle your stock in the best manner, pack it up nicely and give you good count.

When Best to Order-Order now, do not wait until you are ready to plant. Your order will not be shipped until the proper time.

How Best to Remit-Remit by Bank Draft, Express Money Order, or Post Office Money Order on Westminster Post Office, Registered Letter, or stamps for the fractional part of a dollar. Payments invariably in advance.

Prices named in this list supercede all former quotations and are subject to change without notice.

Claims for Deduction should be made within five days from receipt of goods.

This special price list is our agent and the kinds and varieties of stock named herein are comprised largely of the stock we grow.

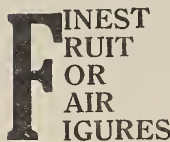

\section{J. E. STONER}

Sole Proprietor of The Westminster Nursery

Westminster, Md.

Long Distance C. \& P. Telephone 222

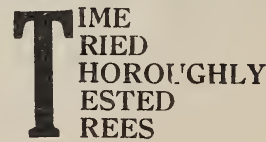

